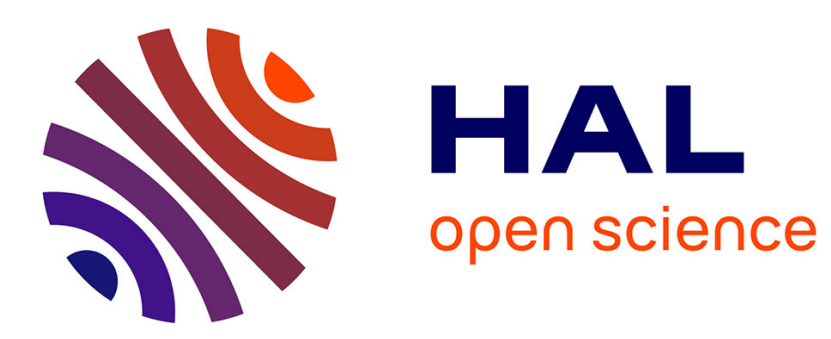

\title{
Size, Albedo, and Taxonomic Type of Potential Spacecraft Target Asteroid (10302) $1989 \mathrm{ML}$
}

Michael Mueller, Alan W. Harris, Alan Fitzsimmons

\section{To cite this version:}

Michael Mueller, Alan W. Harris, Alan Fitzsimmons. Size, Albedo, and Taxonomic Type of Potential Spacecraft Target Asteroid (10302) 1989 ML. Icarus, 2007, 187 (2), pp.611. 10.1016/j.icarus.2007.01.002 . hal-00499068

\section{HAL Id: hal-00499068 https://hal.science/hal-00499068}

Submitted on 9 Jul 2010

HAL is a multi-disciplinary open access archive for the deposit and dissemination of scientific research documents, whether they are published or not. The documents may come from teaching and research institutions in France or abroad, or from public or private research centers.
L'archive ouverte pluridisciplinaire HAL, est destinée au dépôt et à la diffusion de documents scientifiques de niveau recherche, publiés ou non, émanant des établissements d'enseignement et de recherche français ou étrangers, des laboratoires publics ou privés. 


\section{Accepted Manuscript}

Size, Albedo, and Taxonomic Type of Potential Spacecraft Target Asteroid (10302) 1989 ML

Michael Mueller, Alan W. Harris, Alan Fitzsimmons

PII: S0019-1035(07)00042-5

DOI: $\quad$ 10.1016/j.icarus.2007.01.002

Reference: $\quad$ YICAR 8172

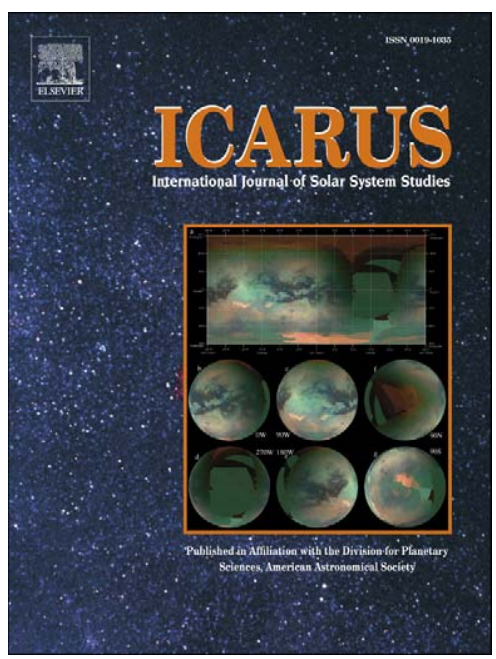

To appear in: Icarus

Received date: 29 September 2006

Revised date: 18 December 2006

Accepted date: 16 January 2007

Please cite this article as: M. Mueller, A.W. Harris, A. Fitzsimmons, Size, Albedo, and Taxonomic Type of Potential Spacecraft Target Asteroid (10302) 1989 ML, Icarus (2007), doi: 10.1016/j.icarus.2007.01.002

This is a PDF file of an unedited manuscript that has been accepted for publication. As a service to our customers we are providing this early version of the manuscript. The manuscript will undergo copyediting, typesetting, and review of the resulting proof before it is published in its final form. Please note that during the production process errors may be discovered which could affect the content, and all legal disclaimers that apply to the journal pertain. 
Size, Albedo, and Taxonomic Type of Potential Spacecraft Target Asteroid (10302) 1989 ML

\title{
Michael Mueller
}

DLR Institute of Planetary Research, Rutherfordstrasse 2, 12489 Berlin, Germany

\section{Alan W. Harris}

DLR Institute of Planetary Research, Rutherfordstrasse 2, 12489 Berlin, Germany

\begin{abstract}
Alan Fitzsimmons
Astrophysics Research Centre, Queen's University Belfast, BT7 1NN, UK
\end{abstract}

Submitted as Note to Icarus on 2006 Sept 29

Revised manuscript, 2006 Dec 8

No. of manuscript pages: 11 (+ 3 for tables and figures)

No. of figures: 2

No. of tables: 1

Running head: Size, albedo, and taxonomic type of (10302) $1989 \mathrm{ML}$

Please send editorial correspondence to:

Michael Mueller

DLR Institute of Planetary Research

Rutherfordstrasse 2

12489 Berlin

Germany

Tel: $\quad$ +493067055406

Fax: $\quad+493067055340$

E-mail: michael.mueller@dlr.de 


\section{Abstract}

The Amor-type near-Earth asteroid (10302) 1989 ML has an "Earth-like" orbit (period $1.44 \mathrm{yr}$, eccentricity 0.14 , inclination $4.4^{\circ}$ ), therefore the energy required to reach it from the Earth is relatively small making it a very attractive target for rendezvous missions. We have observed $1989 \mathrm{ML}$ in the thermal-infrared using the Spitzer Space Telescope, and compared these data with optical and near-infrared observations. The Spitzer results imply a diameter of $0.28 \pm$ $0.05 \mathrm{~km}$ and a geometric albedo of $0.37 \pm 0.15$; together with the reflectance spectrum they are consistent with the relatively rare E classification.

Keywords: Asteroids, Infrared Observations, Near-Earth Objects, Spectrophotometry

\section{Introduction}

The most accessible asteroids for rendezvous missions are those with orbits similar to that of the Earth. Indeed, some near-Earth asteroids (NEAs) are easier to reach than the Moon. The energy and flight time required to reach the NEA (10302) $1989 \mathrm{ML}$ are relatively small, comparable to those for the Hayabusa target (25143) Itokawa (Perozzi et al., 2001;

Christou 2003; Binzel et al., 2004), making it a very attractive spacecraft target for rendezvous missions. $1989 \mathrm{ML}$ has been considered as a possible target for both the Japanese Hayabusa and the European Don Quijote missions (see, for example, Binzel et al., 2001, and http://www.esa.int/SPECIALS/NEO/); at the time of writing further missions to NEAs are being planned in Japan, Europe, and the USA, for which $1989 \mathrm{ML}$ may be considered as a target. However, a serious and urgent problem for mission planning is the lack of information on the physical properties of this asteroid.

Preliminary optical lightcurve measurements by Abe et al. (2000) and Weissman et al. (1999) suggest the peak-to-peak amplitude is about $1 \mathrm{mag}$, corresponding to a very elongated shape. Weissman et al. report a rotation period near $19 \mathrm{~h}$ However, according to Abe et al., $\sim 32 \mathrm{~h}$ is also possible. This long-period high-amplitude lightcurve has limited the accuracy of 
determinations of the absolute optical magnitude $\mathrm{H}$ (see Bowell et al., 1989 for details of the $\mathrm{H}, \mathrm{G}$ magnitude system): Abe et al. (2000) report $\mathrm{H}=19.7$ (assuming $\mathrm{G}=0.15$ ); NEODys (as of 2006/12/08) reports $H=19.39(G=0.15)$; Weissman et al. (1999) report an absolute magnitude in the R-band of $H_{R}=19.14$, which implies $H=19.51$ using $V-R=0.37 \pm 0.03$ (see section 3.2). Since published $\mathrm{H}$ values for NEAs are notoriously unreliable, we adopt the average value of $\mathrm{H}=19.5$ with a conservative uncertainty of \pm 0.3 .

Binzel et al. (2001) report a neutral Xc-type spectrum at optical wavelengths, implying that 1989 ML belongs to one of the $\mathrm{E}, \mathrm{M}$, or $\mathrm{P}$ spectrally degenerate classes. $\mathrm{E}$ type asteroids have a high geometric albedo $p_{v}\left(0.3<p_{v}<0.6\right)$ and may be related to enstatite achondrite meteorites; $\mathrm{M}$ type asteroids have moderate albedos around $0.1-0.2$ and some are probably related to metallic meteorites; $P$ type asteroids have very low albedos $\left(p_{v}<0.1\right)$ and appear to be organic-rich, similar to carbonaceous chondrites (see, e.g. Clark et al., 2004a, b).

Determination of the albedo of an X-type asteroid is therefore very important for constraining its composition.

The sizes of asteroids can be determined from optical and thermal-infrared observations, together with appropriate thermal modeling (e.g. Lebofsky and Spencer, 1989; Harris and Lagerros, 2002; and references therein). The diameter $D$, the geometric albedo $p_{v}$, and the absolute optical magnitude $\mathrm{H}$ are related by (Fowler and Chillemi, 1992):

$$
p_{v}=10^{-H / 2.5}(1329 \mathrm{~km} / \mathrm{D})^{2}
$$

We observed $1989 \mathrm{ML}$ in the thermal infrared using the Infrared Array Camera IRAC (Fazio et al., 2004) onboard the Spitzer Space Telescope, and in the optical using the 2.0-m Faulkes Telescope North. We employed the Near-Earth-Asteroid Thermal Model (NEATM, Harris, 1998) to derive the effective diameter from the Spitzer data. NEATM fits require measurements of the lightcurve-averaged thermal continuum at two or more wavelengths. The diameter and the model parameter $\eta$ are varied to obtain the best fit of the model 
continuum to the observed fluxes. Varying $\eta$ varies the effective color temperature of the model thermal continuum, which allows for the combined effects of thermal inertia, beaming due to roughness, and solar phase angle; note that our Spitzer observations took place at a phase angle of 52.3 degrees. For more detailed discussions of the NEATM see Harris (1998), Delbó and Harris (2002), Harris and Lagerros (2002), and Delbó et al. (2003), and references therein.

\section{Observations and data reduction}

\subsection{Spitzer observations}

1989 ML was observed on 2006 June 2 and 3 with the infrared imaging camera IRAC. IRAC has four filter passbands, referred to as channels $1-4$ in the following, with effective wavelengths of $3.550 \mu \mathrm{m}, 4.493 \mu \mathrm{m}, 5.731 \mu \mathrm{m}$, and $7.872 \mu \mathrm{m}$, respectively, and FWHM of more than $20 \%$ (cf. the IRAC data handbook: http://ssc.spitzer.caltech.edu/irac/dh/). We observed the asteroid six times, each observation providing nearly simultaneous photometry in all 4 IRAC channels with five dither positions, of $30 \mathrm{~s}$ integration time each, per field-of-view. The observation time in each case was about 12 minutes, including dead times for telescope slewing and settling, which is significantly shorter than the asteroid rotation period (see Section 1). There was a time gap of $\sim 3.2 \mathrm{~h}$ between consecutive observations, corresponding to $\sim 60^{\circ}$ in rotational phase assuming the nominal rotation period of $19 \mathrm{hr}$ (Weissman et al, 1999). The observing geometry did not change significantly during our observations: the heliocentric distance was $1.270 \mathrm{AU}$, the distance to Spitzer was $0.891 \mathrm{AU}$, and the solar phase angle, $\alpha$, was 52.3 degrees (source: JPL Horizons System; all values are constant during our Spitzer observations to \pm 2 in the last quoted digit or better).

Correction for most instrument artifacts (including dark current and flatfield) and absolute flux calibration against a set of standard stars (see Fazio et al., 2004) was performed by the automated IRAC data reduction pipeline hosted by the Spitzer Science Center, resulting in 'basic calibrated data' (BCD) image frames. We used the software package MOPEX (see 
Makovoz and Khan, 2005) to mosaic the BCD images, i.e. to co-add them in the asteroid rest frame, rejecting non-co-moving sources such as background stars or cosmic ray hits. The asteroid flux was derived from the resulting mosaic images using standard synthetic aperture photometry procedures. A discussion of the color corrections to the derived flux densities is given in Section 3.1. The mosaics for observations $1-5$ display clear asteroid signals at the predicted positions, but observation \#6 failed, because the target asteroid was within 2" of a background star of comparable brightness. Also, data from observation \#4 are compromised by the presence of a faint background source, which can be neglected for IRAC channels 3 and 4 but has comparable flux to that of the asteroid at shorter wavelengths.

The highest signal-to-noise ratio is obtained in IRAC channel 4, from which photometry can be extracted for observations 1 through 5 , yielding a coarse thermal lightcurve. For all channels we stacked images from observations $1-5$ and derived an average flux value from the resulting mosaic images. The flux values are listed in Table 1.

\subsection{Optical observations}

In order to provide further photometric data on $1989 \mathrm{ML}$, it was observed on 2006 January 4.5 UT using the 2.0-m Faulkes Telescope North at the Haleakala observatory on Maui, Hawaii, at a solar phase angle around 1.1 degrees. BVRI imaging was obtained with the DILCAM CCD camera, a clone of the RATCAM instrument (Steele, 2001). The telescope was tracked at sidereal rates, so exposure times were limited to 40 seconds to ensure that trailing of the target did not occur. The NEA was imaged using the sequence R-B-R-V-R-I-R..., to allow interpolation of the colors relative to R over the lightcurve. Unfortunately the observations spanned only 2.5 hours, and so did not allow further refinement of the spin period.

Observations of standard stars (Landolt, 1992) were obtained during this period to allow photometric calibration and hence provide apparent magnitudes and colors.

\section{Results}




\subsection{Spitzer results}

Our time-resolved channel-4 data $(7.872 \mu \mathrm{m})$ given in Table 1 (upper part) are consistent with a high-amplitude long-period lightcurve (see Section 1). Sinusoidal double-peaked lightcurves corresponding to the two possible rotation periods of 19 and $32 \mathrm{~h}$ fit the data well. The average flux levels of the two fitted lightcurves are consistent with one another and with the flux value obtained from stacking images from observations 1 - 5 (cf. Table 1, lower part). Agreement is to within a few percent, a negligible difference compared to the statistical flux uncertainty. We cannot further constrain the rotation properties from our Spitzer data, but conclude that for all IRAC channels the flux values obtained from stacking observations $1-5$ are good proxies to the lightcurve average flux level.

IRAC is a broad-band photometer, so the derived flux values must be color corrected, taking account of the detector spectral response and the asteroid spectral shape. Color correction factors for several target spectra are tabulated in the IRAC data handbook, p. 48 (http://ssc.spitzer.caltech.edu/irac/dh/). A routine was developed to calculate color correction factors for several model spectra, including NEATM spectra (using the IRAC spectral detector responses provided online by the Spitzer Science Center). We verified that our software reproduces the correction factors provided in the data handbook. Due to their different spectral shapes, different color corrections apply to the thermally emitted flux component and to reflected sunlight; color corrections to the latter are negligible.

We estimated the amount of reflected sunlight in channels $1-4$ assuming a solar black body temperature of $5800 \mathrm{~K}$ and a relative reflectance of $\sim 1.2$ between $3.6 \mu \mathrm{m}$ and the $\mathrm{V}$ band (cf. Fig. 2). Reflected sunlight was found to contribute virtually all the measured flux in channel 1 , only negligible amounts in channel 4 , and $\sim 1 \mu \mathrm{Jy}$ in channel 3 ( $\sim 5 \%$ of the measured flux). The relative contributions to the channel 2 flux cannot be easily determined. 
We fitted NEATM to the thermal flux values from channels 3 and 4 and calculated color correction factors using the model parameters (diameter, $p_{v}$, and $\eta$ ) derived. We repeated this procedure with color-corrected thermal fluxes, after which the procedure was seen to have converged; a second iteration brought about changes significantly below the $1 \%$ level. The resulting model spectrum with the observational data overlaid is shown in Figure 1.

The best-fit parameters are: Diameter $D=0.276 \mathrm{~km}$, albedo $p_{v}=0.37$, and $\eta=2.48$. Color corrected fluxes were obtained by dividing the thermal contributions to fluxes by 1.129 (channel 2), 1.070 (channel 4) and 1.034 (channel 4). Uncertainties in D, $p_{v}$ and $\eta$ were estimated using a Monte-Carlo analysis. To this end, we generated a random set of synthetic thermal flux values at the channel-3 and channel-4 wavelengths, normally distributed around the measured values, and fitted them with NEATM. We rejected unrealistic results with $\eta>3$ or $p_{V}>0.7$. The remaining sample of 15,000 results gave $D=0.246 \pm 0.037 \mathrm{~km}, p_{V}=0.46 \pm$ 0.13 , and $\eta=2.23 \pm 0.44$ ( $1 \sigma$ standard deviations - note, however, that the distribution of resulting model parameters is highly non-Gaussian). We adopt the relative uncertainties from this simulation for our best-fit results stated above, yielding: $D=0.276 \pm 0.041 \mathrm{~km}, \mathrm{p}_{V}=0.37 \pm$ 0.11 , and $\eta=2.48 \pm 0.49$. The uncertainty in our adopted value for $H(19.5 \pm 0.3)$ contributes an additional $30 \%$ to the albedo error (added in quadrature) so $p_{v}=0.37 \pm 0.15$. This albedo is suggestive of an E classification. $P$ types, for which $p_{v}$ should not exceed 0.1 (cf. Section 1), would appear to be ruled out (see also Fig. 1). We note that in addition to the statistical errors there is a systematic modeling uncertainty that increases with solar phase angle and thermal inertia (see Harris, 2006), but in the sense of underestimating $p_{v}$. So the systematic uncertainty in the NEATM results in this case would tend to increase $p_{\vee}$ above the value of 0.37 derived here.

The derived $\eta$ value of $2.5 \pm 0.5$ is rather high for a solar phase angle of $52^{\circ}$ (Delbó et al., 2003) and is consistent with a high surface thermal inertia, corresponding to a lack of 
thermally insulating dust or regolith. Better spectral coverage and a thermophysical model would be required to derive conclusive statements about surface thermal properties.

\subsection{Optical and near-infrared data}

Our optical photometry revealed the following colors: $(B-V)=0.63 \pm 0.05,(V-R)=0.37 \pm 0.03$, $(V-I)=0.74 \pm 0.03$. These colors are consistent with those reported by Abe et al. (2000) but have significantly smaller uncertainties. ECAS and near-IR (JHK) photometry of $1989 \mathrm{ML}$ has also been obtained by D. Tholen and is reported in Hiroi et al. (2000). Taken together, these datasets show that the optical/NIR colors are redder than solar, with a spectral break to a less steep slope occurring around a wavelength of $\sim 0.7$ microns; they are consistent with the optical spectrum reported by Binzel et al. (2001).

To investigate the possible taxonomic types we compared these data with spectra of previously classified asteroids from the SMASS II (Bus and Binzel, 2003) and the 52-color catalogues (Bell et al., 1995). Figure 2 shows the optical and IR photometry compared to 52color data of the E-type asteroid (44) Nysa, demonstrating a clear correspondence. However, the colors of the E-type asteroids (64) Angelina and (3101) Eger are inconsistent with the 1989 ML colors. Clark et al. (2004a) have shown that Nysa-type spectra are consistent with a silicate mineralogy higher in iron than the mineral enstatite, while Angelina-type spectra are consistent with sulfide-bearing silicates. Interestingly, although E, M and P-types are spectrally degenerate in the optical, we were not able to obtain similar agreement with 52color data of the M-types (16) Psyche and (216) Kleopatra, nor the P-types (65) Cybele and (153) Hilda. Therefore we conclude that the available optical-NIR data support a relatively Feenriched E-type classification, in agreement with our Spitzer data.

\section{Summary}

On the basis of thermal-infrared photometric observations of near-Earth asteroid (10302) 1989 ML using the IRAC camera onboard the Spitzer Space Telescope we have determined its 
effective diameter to be $0.28 \pm 0.05 \mathrm{~km}$ and its geometric albedo $p_{v}$ to be $0.37 \pm 0.15$. This high albedo is incompatible with a P-type classification, is only marginally consistent with an M-type classification, but is fully consistent with an E-type classification. The available optical and near-infrared data also favor E type. Taken together, we conclude that the available data suggest an E classification for (10302) $1989 \mathrm{ML}$ - note that only 4 E-type near-Earth asteroids were known so far (Clark et al., 2004a).

\section{Acknowledgments}

This work is based on observations made with the Spitzer Space Telescope, which is operated by the Jet Propulsion Laboratory, California Institute of Technology under a contract with NASA. The comments and suggestions of the referees, Beth Clark and Marco Delbó, are gratefully acknowledged.

\section{References}

Abe, M., Sato, I., Araki, H., 2000. Lightcurve and color of near-Earth-asteroid 1989ML. Adv. Space Res. 25, $269-272$.

Bell, J. F., Owensby, P. D., Hawke, B. R., Brown, R. H., Cruikshank, D., and Hartmann, W. K., 1995. 52-color Asteroid Survey. EAR-A-RDR-3-52COLOR-V2.1. NASA Planetary Data System.

Binzel, R. P., Harris, A. W., Bus, S. J., Burbine, T. H. 2001. Spectral properties of near-Earth objects: Palomar and IRTF results for 48 objects including spacecraft targets (9969) Braille and (10302) $1989 \mathrm{ML}$. Icarus 151, 139 - 149.

Binzel, R. P., Perozzi, E., Rivkin, A. S., Rossi, A., Harris, A. W., Bus, S. J., Valsecchi, G. B., Slivan, S. M., 2004. Dynamical and compositional assessment of near-Earth object mission targets. Meteoritics and Plan. Sci. 39, 351 - 366.

Bowell, E., Hapke, B., Domingue, D., Lumme, K., Peltoniemi, J., Harris, A. W., 1989. Application of photometric models to asteroids. In: R. P. Binzel et al. (Eds.), Asteroids II, Univ. of Arizona Press, Tucson, pp. $524-556$. 
Bus, S. and Binzel, R. P., 2003. Small Main-belt Asteroid Spectroscopic Survey, Phase II. EAR-A-I0028-4-SBN0001/SMASSII-V1.0. NASA Planetary Data System.

Christou, A. A. 2003. The statistics of flight opportunities to accessible near-Earth asteroids. Planet. Space Sci. 51, $221-231$.

Clark, B. E., Bus, S. J., Rivkin, A. S., McConnochie, T., Sanders, J., Shah, S., Hiroi, T., Shepard, M., 2004a. E-type asteroid spectroscopy and compositional modeling. J. Geophys. Res. 109, E2, E02001.

Clark, B. E., Bus, S. J., Rivkin, A. S., Shepard, M. K., Shah, S. 2004b. Spectroscopy of XType Asteroids. Astron. J. 128, 3070 - 3081.

Delbó, M., Harris, A. W., 2002. Physical properties of near-Earth asteroids from thermal infrared observations and thermal modeling. Meteoritics and Plan. Sci. 37, 1929-1936.

Delbó, M., Harris, A. W., Binzel, R. P, Pravec, P., Davies, J. K., 2003. Keck observations of near-Earth asteroids in the thermal infrared. Icarus 166, 116-130.

Fazio, G. G. and 64 colleagues, 2004. The Infrared Array Camera (IRAC) for the Spitzer Space Telescope. Astrophys. J. Suppl. 154, $10-17$.

Fowler, J. W. and Chillemi, J. R., 1992. IRAS data processing. In: Tedesco, E.F. (Ed.), The IRAS Minor Planet Survey. Tech. Rpt. PL-TR-92-2049. Phillips Laboratory, Hanscom Air Force Base, Massachusetts, pp. 17 - 43.

Harris, A. W. 1998. A thermal model for near-Earth asteroids. Icarus 131, 291 - 301.

Harris, A. W., 2006. The surface properties of small asteroids from thermal-infrared observations. In: Lazzaro, D., Ferraz-Mello, S., Fernández, J. A. (Eds.), Proc. of IAU Symposium 229. Cambridge University Press, Cambridge, pp. 449 - 463.

Harris, A. W., and Lagerros, J.S.V., 2002. Asteroids in the thermal infrared. In: Bottke, W.F., Paolicchi, P., Binzel, R.P., Cellino, A. (Eds.), Asteroids III. Univ. of Arizona Press, Tucson, pp. $205-218$.

Hiroi, T., Zolensky, E. M., Lipschutz, E. M., 2000. Possible meteorite analogs for asteroid 1989ML - Target of MUSES-C asteroid sample return mission. Antarctic Meteorites 25, $14-15$. 
Landolt, A. U., 1992. UBVRI photometric standard stars in the magnitude range $11.5-16.0$ around the celestial equator. Astron. J. 104, $340-371,436-491$.

Lebofsky, L. A., Spencer, J. R., 1989. Radiometry and thermal modeling of asteroids. In: Binzel, R.P., Gehrels, T., Matthews, M. S., (Eds.), Asteroids II. Univ. of Arizona Press, Tucson, pp. $128-147$.

Makovoz, D. and Khan, I., 2005. Mosaicking with MOPEX. In: Shopbell, P. L., Britton, M. C., Ebert, R. (Eds.), Astronomical Data Analysis Software and Systems XIV ASP Conf. Ser. 347, ASP, San Francisco, pp. $81-85$.

Perozzi, E., Rossi, A., Valsecchi, G.B., 2001. Basic targeting strategies for rendezvous and flyby missions to the near-Earth asteroids. Planet. Space Sci. 49, 3- 22.

Steele, I. A., 2001. The Liverpool Telescope. Astron. Nachr. 332, 307 - 310.

Weissman, P., Doressoundiram, A., Hicks, M., Chamberlin, A., Sykes, M., Larson, S., Hergenrother, C., 1999. CCD Photometry of Comet and Asteroid Targets of Spacecraft Missions. DPS meeting \#31, \#30.03. 
Table 1

Flux densities derived from Spitzer IRAC observations. Above: Time-resolved channel-4 fluxes $(7.872 \mu \mathrm{m})$. Below: Average fluxes for all four IRAC channels derived from stacking observations $1-5$.

\begin{tabular}{lll}
\hline JD -2453889.0 & Flux $(\mu \mathrm{Jy})$ & $\sigma$ flux $(\mu \mathrm{Jy})$ \\
\hline 0.47827 & $126(122)$ & $19(18)$ \\
0.60011 & $49(47)$ & $16(15)$ \\
0.75043 & $115(112)$ & $23(22)$ \\
0.86787 & $188(182)$ & $17(16)$ \\
1.01833 & $91(89)$ & $21(20)$ \\
\hline & & $\sigma$ flux $(\mu \mathrm{Jy})$ \\
\hline Central wavelength $(\mu \mathrm{m})$ & Flux $(\mu \mathrm{Jy})$ & $0.77(0.77)$ \\
\hline 3.550 & $4.14(4.14)$ & $1.3(1.2)$ \\
5.493 & $4.1 \quad(3.8)$ & $7.3(6.8)$ \\
7.872 & $21.7(20.3)$ & $17.7(18)$ \\
\hline
\end{tabular}

Notes. Flux values in parentheses are color corrected (see Section 3.1). Times refer to the beginning of the observations and are not light-time corrected. 
Figure Captions

Figure 1: Model fits to the photometric data given in Table 1. Both reflected sunlight and thermal emission are modeled (cf. text). The thermal contributions to channels 3 and 4 (wavelengths 5.7 and $7.9 \mu \mathrm{m}$ ) are fitted using NEATM; the reflected sunlight is extrapolated from the predicted $V$ magnitude assuming a relative reflectance of 1.2 (cf. text and Fig. 2). An albedo of $p_{v}=0.05$, which would be typical for P-type asteroids, is clearly incompatible with the data (dashed line).

Figure 2: Comparison of photometry of $1989 \mathrm{ML}$ with spectra of (44) Nysa. Square symbols represent optical colors given in this paper; circles represent the optical and near-infrared data reported by Hiroi et al. (2000). The continuous line covering $0.4-1.0$ micron is the spectrum of (44) Nysa from the SMASS II dataset by Bus and Binzel (2003). The three continuous lines extending from 0.9 to 2.5 microns are individual spectra of (44) Nysa from the 52-color asteroid survey (Bell et al., 1995). Optical data were normalized to a wavelength of $0.55 \mu \mathrm{m}$. The near-infrared spectra were scaled to match the optical spectrum near $0.95 \mu \mathrm{m}$. 
Figure 1:

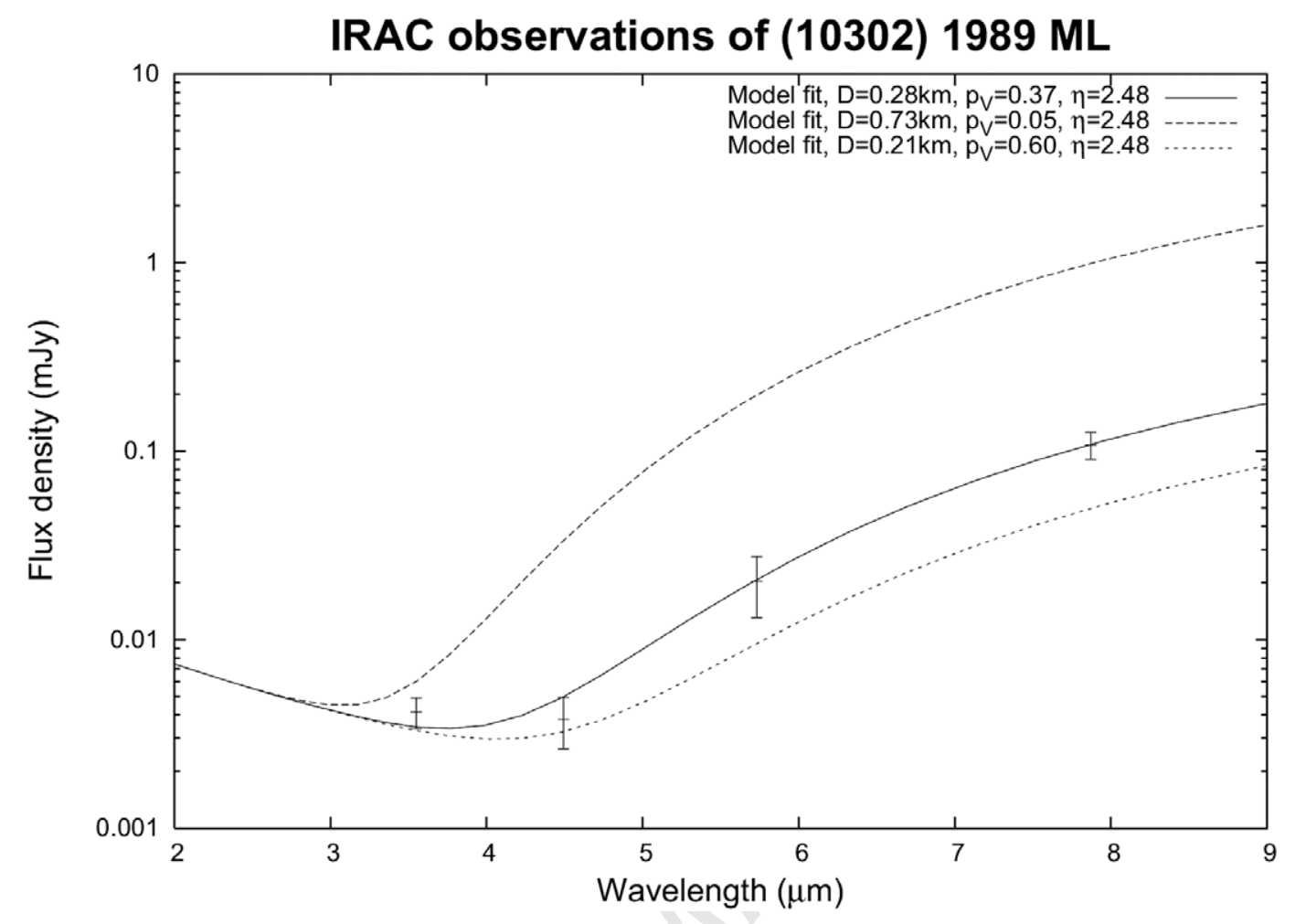

Figure 2:

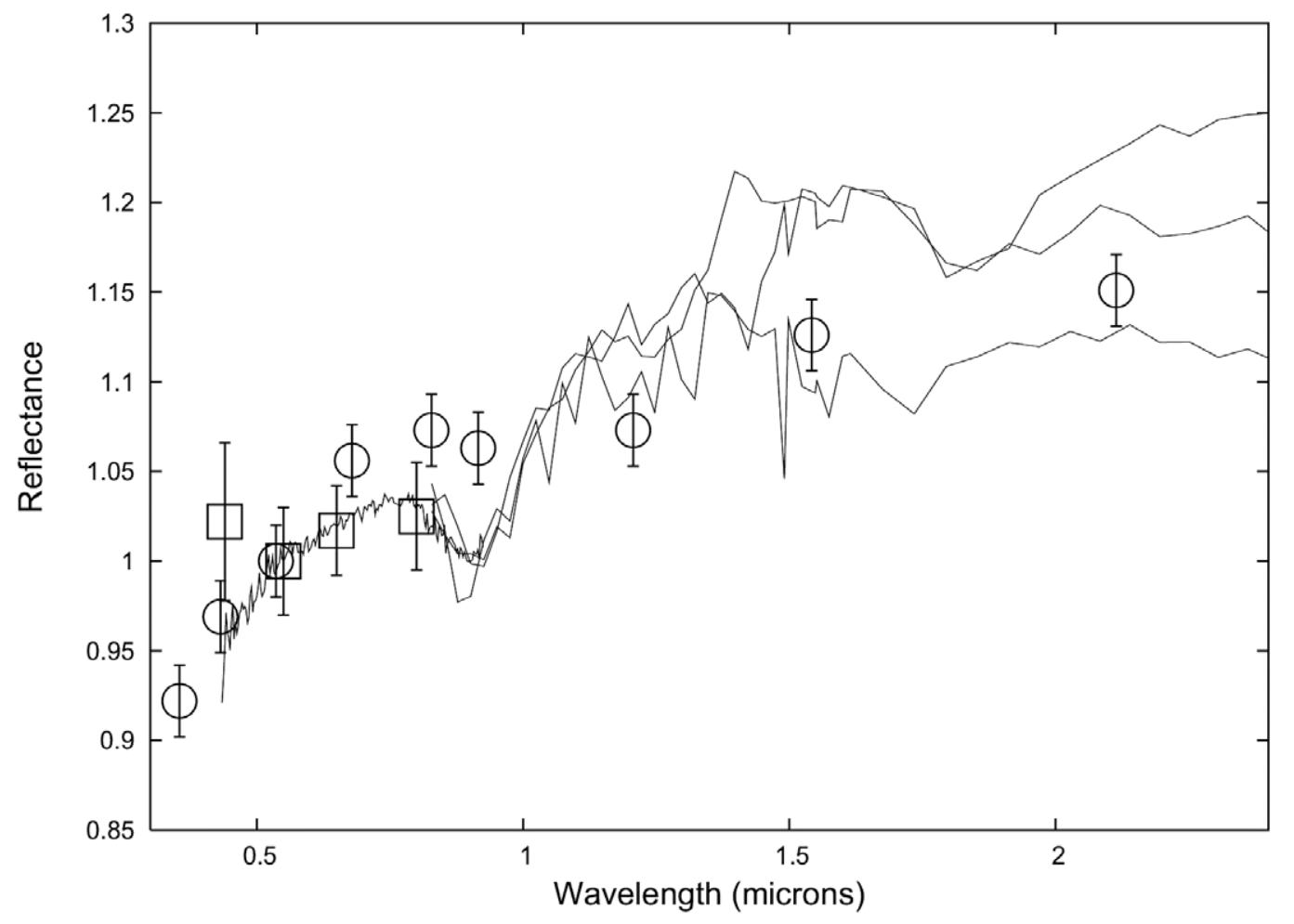

